\title{
One-loop correction to the AdS/BCFT partition function in three-dimensional pure gravity
}

\author{
Yu-ki Suzuki ${ }^{*}$ \\ Center for Gravitational Physics, Yukawa Institute for Theoretical Physics, Kitashirakawa Oiwakecho, \\ Sakyo-ku, Kyoto University, Kyoto 606-8502, Japan
}

(Received 28 July 2021; accepted 10 January 2022; published 25 January 2022)

\begin{abstract}
We calculate the tree-level partition function of a Euclidean Bañados-Teitelboim-Zanelli (BTZ) black hole in the presence of end of the world branes (ETW branes) and the one-loop partition function of a Euclidean thermal $\mathrm{AdS}_{3}$ in the presence of an ETW brane. At the tree level, our results match with the previous ones for static BTZ black holes. Interestingly, at the one-loop level, our results contain novel terms, which reflect the existence of an ETW brane. The ETW brane has a consistent profile for a static thermal AdS and, in this case, we showed that the spectrum obtained from the one-loop partition function takes a physically sensible form.
\end{abstract}

DOI: 10.1103/PhysRevD.105.026023

\section{INTRODUCTION}

The one-loop partition function in gravity was calculated almost half a century ago in the famous work [1]. In the context of the AdS/CFT correspondence [2], there have been several works toward deriving the full quantum gravity partition function of pure gravity at a finite temperature [3-5]. At the one-loop level, they deduced the result from the CFT path integral calculation [4], and later this was directly proved using the heat kernel method [3], summarized into the formula,

$$
Z_{\text {gravity }}=\prod_{m=2}^{\infty} \frac{1}{\left|1-q^{m}\right|^{2}},
$$

where $q$ describes the moduli of the boundary torus.

Our goal is to extend this calculation to the AdS/BCFT case. The AdS/BCFT correspondence is an extension of the AdS/CFT correspondence to the case where a CFT lives on a manifold with boundaries (called boundary conformal field theory, or BCFT) [6-9]. The basic idea of the AdS/ BCFT is to extend the boundary of the manifold where the CFT is defined, to a codimension one surface in the AdS, called the end of the world brane (ETW brane). The gravity in the spacetime, surrounded by this ETW brane, provides the gravity dual of the BCFT. In this paper, first, we will calculate the tree-level partition function of a rotating

\footnotetext{
’yu-ki.suzuki@yukawa.kyoto-u.ac.jp
}

Published by the American Physical Society under the terms of the Creative Commons Attribution 4.0 International license. Further distribution of this work must maintain attribution to the author(s) and the published article's title, journal citation, and DOI. Funded by SCOAP ${ }^{3}$.
Euclidean Bañados-Teitelboim-Zanelli (BTZ) black hole in the presence of ETW branes with an arbitrary tension, which is summarized as the result,

$$
\begin{aligned}
Z_{\mathrm{BTZ}-\text { tree }}= & \exp \left[\frac{\pi R_{+}}{8 G}+\frac{1}{8 G}\left(\log \left(\frac{1+T_{\pi}}{1-T_{\pi}}\right)\right.\right. \\
& \left.\left.-\log \left(\frac{1+T_{0}}{1-T_{0}}\right)\right)\right] .
\end{aligned}
$$

In the static (i.e., nonrotating) case, this matches with the previous calculation [6]. Secondly, we will calculate the one-loop partition function of a thermal $\mathrm{AdS}_{3}$ in the presence of a tensionless ETW brane with the Neumann boundary condition (for earlier discussions of the boundary conditions in gravity refer to $[10,11])$. Our final result is given by

$Z_{\text {gravity }}=\prod_{m=2}^{\infty} \frac{1}{\left|1-q^{m}\right|} \cdot \prod_{l=0}^{\infty} \frac{\sqrt{1-q^{l+2} \bar{q}^{l+1}} \sqrt{1-q^{l+1} \bar{q}^{l+2}}}{\sqrt{1-q^{l+2} \bar{q}^{l}} \sqrt{1-q^{l} \bar{q}^{l+2}}}$.

There are two contributions: the first term corresponds to a square root of the original result, which comes from the bulk modes, and the second term is the new effect due to the ETW brane. The second term represents the contributions from a massive ghost vector field, due to the Becchi-RouetStora-Tyutin (BRST) quantization of gravity, and the massless spin-2 field. However, as we consider later, if we set $q=\bar{q}$ to have a consistent configuration of ETW brane, then we have a physically sensible partition function with non-negative coefficients in the $q$ expansion like (1).

One might wonder if we can impose the Dirichlet boundary condition on the ETW brane [12]. At the tree 
level, both the Neumann and the Dirichlet boundary condition lead to an identical partition function for a tensionless ETW brane. On the other hand, at the oneloop level, the resulting partition function for the Dirichlet boundary condition is different from that for the Neumann one. However, as we will see later, we find that the Dirichlet boundary condition is in tension with the BRST invariance.

Finally, we will discuss summing over the modular transformation. The boundary CFT lives on the conformal boundary of a half of the solid torus [13] in our AdS/BCFT setup. We expect that it still admits modularity even if we insert branes because from the boundary torus perspective it still admits modular invariance, and then we consider how to locate the position of the brane in the bulk. To derive the full partition function, we must sum over the $S L(2, \mathbb{Z})$ transformation of the thermal AdS contribution $[4,14]$.

This paper is organized as follows. In Sec. II we directly calculate the partition function of a rotating BTZ black hole with ETW branes. There are already earlier works on this calculation in the no boundary case [15-18]. In Sec. III, we review the heat kernel method for calculating a one-loop partition function [19-22], and then we apply this to the computation of a one-loop partition function of the scalar, the vector, and the spin-2 fields using a method of images in the AdS/BCFT. In Sec. IV, we discuss the physical interpretation of the partition function and the consistency of the boundary conditions. In Sec. V we discuss our conclusions and present some future directions. In Appendix A we give the detailed calculation of derivatives of chordal distance $u$. In Appendix B, we present the calculation of a partition function in the Dirichlet case.

\section{A TREE-LEVEL PARTITION FUNCTION OF A BTZ BLACK HOLE WITH ETW BRANES}

\section{A. BTZ with tensionless ETW branes}

Let us consider a Euclidean rotating BTZ black hole in three dimension [15]. The metric is given by

$$
\begin{aligned}
d s^{2}= & \frac{\left(r^{2}-R_{+}^{2}\right)\left(r^{2}+R_{-}^{2}\right)}{r^{2}} d t^{2}+\frac{r^{2}}{\left(r^{2}-R_{+}^{2}\right)\left(r^{2}+R_{-}^{2}\right)} d r^{2} \\
& +r^{2}\left(d \phi-\frac{R_{+} R_{-}}{r^{2}} d t\right)^{2} .
\end{aligned}
$$

Here $R_{-}$is a real valued parameter, and $R_{+}$is the horizon radius. The absence of a conical singularity constrains the periodicity of time and rotational angle,

$$
(t, \phi) \sim(t+\beta, \phi+\theta),
$$

where $\beta=\frac{2 \pi R_{+}}{R_{+}^{2}+R_{-}^{2}}$ and $\theta=\frac{2 \pi R_{-}}{R_{+}^{2}+R_{-}^{2}}$. If we define the new coordinate as $\phi^{\prime} \equiv \phi-\frac{\theta}{\beta} t$, then the periodicity can be recast as $\left(t, \phi^{\prime}\right) \sim\left(t+\beta, \phi^{\prime}\right)$ [16]. We also rewrite the metric in terms of this coordinate as

$$
g_{a b}=\left(\begin{array}{ccc}
A & 0 & B \\
0 & C & 0 \\
D & 0 & E
\end{array}\right),
$$

where

$$
\begin{aligned}
& A=\frac{\left(r^{2}-R_{+}^{2}\right)\left(r^{2}+R_{-}^{2}\right)}{r^{2}}+r^{2}\left(\frac{R_{-}}{R_{+}}-\frac{R_{+} R_{-}}{r^{2}}\right)^{2}, \\
& B=\frac{R_{-}}{R_{+}} r^{2}-R_{+} R_{-}, \\
& C=\frac{r^{2}}{\left(r^{2}-R_{+}^{2}\right)\left(r^{2}+R_{-}^{2}\right)}, \\
& D=\frac{R_{-}}{R_{+}} r^{2}-R_{+} R_{-}, \\
& E=r^{2} .
\end{aligned}
$$

Note that $\operatorname{det}\left(g_{i j}\right)=r^{2}$. To identify the location of an ETW brane, we use the map to the Poincare coordinate,

$$
\begin{aligned}
& \eta=\left(\frac{r^{2}-R_{+}^{2}}{r^{2}+R_{-}^{2}}\right)^{\frac{1}{2}} \cos \left(\frac{R_{+}^{2}+R_{-}^{2}}{R_{+}} t+R_{-} \phi^{\prime}\right) \exp \left(R_{+} \phi^{\prime}\right), \\
& x=\left(\frac{r^{2}-R_{+}^{2}}{r^{2}+R_{-}^{2}}\right)^{\frac{1}{2}} \sin \left(\frac{R_{+}^{2}+R_{-}^{2}}{R_{+}} t+R_{-} \phi^{\prime}\right) \exp \left(R_{+} \phi^{\prime}\right), \\
& z=\left(\frac{R_{+}^{2}+R_{-}^{2}}{r^{2}+R_{-}^{2}}\right)^{\frac{1}{2}} \exp \left(R_{+} \phi^{\prime}\right)
\end{aligned}
$$

which leads to the familiar metric

$$
d s^{2}=\frac{d z^{2}+d x^{2}+d \eta^{2}}{z^{2}} .
$$

In this coordinate the identification $\left(t, \phi^{\prime}\right) \sim\left(t+\beta, \phi^{\prime}\right)$ is trivial, but the identification $\left(t, \phi^{\prime}\right) \sim\left(t, \phi^{\prime}+2 \pi\right)$ is nontrivial. If we define the following complexified coordinate $w=\eta+i x$, then the identification is translated into $(w, z) \sim\left(w e^{2 \pi\left(R_{+}+i R_{-}\right)}, z e^{2 \pi R_{+}}\right)$. From this perspective, we can take the fundamental region as $1 \leq|w|^{2}+z^{2} \leq e^{2 \pi R_{+}}$, and the horizon $r=R_{+}$is mapped to $w=0$ and $1 \leq z \leq e^{2 \pi R_{+}}$.

Let us insert the ETW branes in this setup. The position of ETW branes with a tension $T$ is determined by the Neumann boundary condition in the AdS/BCFT,

$$
K_{a b}-K h_{a b}=-T h_{a b} .
$$

In the Poincare coordinate, the solution to the boundary condition (10) is solved as follows [23]:

$$
(z-\alpha)^{2}+(x-p)^{2}+(\eta-q)^{2}=\beta^{2},
$$


where the tension is given by $T=\frac{\alpha}{\beta}$. Here ETW branes, where $\phi^{\prime}$ is constant, are just a sphere of radius 1 for $\phi^{\prime}=0$ and $e^{\pi R_{+}}$for $\phi^{\prime}=\pi$. Next, we calculate the action

$$
\begin{aligned}
S= & -\frac{1}{16 \pi G} \int_{N} \sqrt{g}(R+2) d^{3} x-\frac{1}{8 \pi G} \int_{Q} \sqrt{h}(K-T) d^{2} x \\
& -\frac{1}{8 \pi G} \int_{M} \sqrt{h} K d^{2} x+\frac{k}{8 \pi G} S_{c t},
\end{aligned}
$$

where $N$ is the three-dimensional bulk region, $Q$ is the ETW brane, and $M$ is the conformal boundary placed at $r=R$, which we later take $R \rightarrow \infty$. Note that $S_{c t}=\int_{M} \sqrt{h}$ is a counterterm constructed only from induced geometric quantities [24].

Consider computing the induced metric and the extrinsic curvature at $r=R$ surface. After some algebras, we get

$$
h_{a b}=\left(\begin{array}{ccc}
A^{\prime} & 0 & B^{\prime} \\
0 & 0 & 0 \\
B & 0 & C^{\prime}
\end{array}\right)
$$

where

$A^{\prime}==\frac{\left(r^{2}-R_{+}^{2}\right)\left(r^{2}+R_{-}^{2}\right)}{R^{2}}+R^{2}\left(\frac{R_{-}}{R_{+}}-\frac{R_{+} R_{-}}{R^{2}}\right)^{2}$,

$B^{\prime}=\frac{R_{-}}{R_{+}} R^{2}-R_{+} R_{-}$,

$C^{\prime}=R^{2}$,

and the extrinsic curvature reads

$$
K_{a b}=\left(\begin{array}{cc}
\frac{R_{+}^{2}+R_{-}^{2}}{R_{+}^{2}} & \frac{R_{-}}{R_{+}} \\
\frac{R_{-}}{R_{+}} & 1
\end{array}\right) \sqrt{\left(R^{2}-R_{+}^{2}\right)\left(r^{2}+R_{-}^{2}\right)} .
$$

From this, we find

$$
\begin{aligned}
\operatorname{det}\left(h_{a b}\right) & =\left(R^{2}-R_{+}^{2}\right)\left(R^{2}+R_{-}^{2}\right), \\
K & =\frac{2 R^{2}-R_{+}^{2}+R_{-}^{2}}{\sqrt{\left(R^{2}-R_{+}^{2}\right)\left(R^{2}+R_{-}^{2}\right)}} .
\end{aligned}
$$

Now, the Einstein-Hilbert action is just the volume integral,

$$
-\frac{1}{16 \pi G} \int_{R_{+}}^{R} d r \int_{0}^{\beta} d t \int_{0}^{\pi} d \phi^{\prime}(-4 r)=\frac{4 \pi \beta}{16 \pi G}\left(\frac{R^{2}-R_{+}^{2}}{2}\right) .
$$

We can also evaluate the Gibbons-Hawking-York $(G H Y)$ term at $r=R$,

$$
\begin{aligned}
& -\frac{1}{8 \pi G} \int_{0}^{\beta} d t \int_{\frac{2 \pi}{\beta}\left(1-\frac{t}{\beta}\right)}^{\frac{2 \pi}{\beta}\left(1-\frac{t}{)}\right)+\pi} d \phi^{\prime}\left[\sqrt{\left(R^{2}-R_{+}^{2}\right)\left(R^{2}+R_{-}^{2}\right)}\right. \\
& \left.\quad \times\left(\frac{2 R^{2}-R_{+}^{2}+R_{-}^{2}}{\left(R^{2}-R_{+}^{2}\right)\left(R^{2}+R_{-}^{2}\right)}\right)\right]=-\frac{\beta}{8 G}\left(2 R^{2}-R_{+}^{2}+R_{-}^{2}\right) .
\end{aligned}
$$

Then, we determine the counterterm. That should be constructed from a geometric quantity of the boundary surface. Here, as the most simple one $(k=1)$, we take

$$
S_{c t}=\int_{M} d^{2} x \sqrt{h}=\beta \pi \sqrt{\left(R^{2}-R_{+}^{2}\right)\left(R^{2}+R_{-}^{2}\right)} .
$$

We combine the above results altogether, and we get

$$
S=-\frac{\beta}{8 G}\left(R^{2}+R_{-}^{2}-\sqrt{\left(R^{2}-R_{+}^{2}\right)\left(R^{2}+R_{-}^{2}\right)}\right) .
$$

If we take $R \rightarrow \infty$ limit, then this becomes

$$
S \simeq-\frac{\beta\left(R_{+}^{2}+R_{-}^{2}\right)}{16 G}=-\frac{\pi R_{+}}{8 G}=-\frac{\pi^{2} \beta}{4 G\left(\beta^{2}+\theta^{2}\right)} .
$$

This is what we expected because we chose the position of the ETW branes so that the volume of the bulk space becomes a half of the original volume. In the full AdS case, this is done in [18], which is just twice of the action in our calculation.

\section{B. BTZ with ETW branes for general values of tension $T$}

Next, we consider the general case with nonvanishing $T$. It is natural to assume the rotational symmetry, i.e., setting $p=q=0$ in (11). Therefore, we will consider the following equation:

$$
\left(\left(\frac{R_{+}^{2}+R_{-}^{2}}{r^{2}+R_{-}^{2}}\right)^{\frac{1}{2}} e^{R_{+} \phi^{\prime}}-\alpha\right)^{2}+\left(\frac{r^{2}-R_{+}^{2}}{r^{2}+R_{-}^{2}}\right) e^{2 R_{+} \phi^{\prime}}=\beta^{2} .
$$

Since the brane is anchored at $\phi^{\prime}=0$ and $\pi$, if we take $r \rightarrow \infty$, then the brane equation becomes

$$
\begin{aligned}
& \alpha^{2}+1=\beta^{2} \quad\left(\phi^{\prime}=0\right), \\
& \alpha^{2}+\exp \left(2 \pi R_{+}\right)=\beta^{2} \quad\left(\phi^{\prime}=\pi\right) .
\end{aligned}
$$

We note that $T$ has range $-1<T<1$ from the above constraint. Using this, we can determine the brane configuration; in the case where the brane is anchored at $\phi^{\prime}=0$, the equation becomes 


$$
\begin{aligned}
\phi^{\prime}= & \frac{1}{R_{+}} \log \left(\frac{T}{\sqrt{1-T^{2}}} \sqrt{\frac{R_{+}^{2}+R_{-}^{2}}{r^{2}+R_{-}^{2}}}\right. \\
& \left.+\sqrt{\frac{T^{2}}{1-T^{2}}\left(\frac{R_{+}^{2}+R_{-}^{2}}{r^{2}+R_{-}^{2}}\right)+1}\right),
\end{aligned}
$$

and in the case where the brane is anchored at $\phi^{\prime}=\pi$, we have

$$
\begin{aligned}
\phi^{\prime}= & \frac{1}{R_{+}} \log \left\{e ^ { \pi R _ { + } } \left(\frac{T}{\sqrt{1-T^{2}}} \sqrt{\frac{R_{+}^{2}+R_{-}^{2}}{r^{2}+R_{-}^{2}}}\right.\right. \\
& +\sqrt{\left.\left.\frac{T^{2}}{1-T^{2}}\left(\frac{R_{+}^{2}+R_{-}^{2}}{r^{2}+R_{-}^{2}}\right)+1\right)\right\} .}
\end{aligned}
$$

Then the Einstein-Hilbert action can be calculated directly,

$$
\begin{aligned}
S_{\mathrm{EH}} & =-\frac{1}{16 \pi G} \int_{R_{+}}^{R_{\mathrm{reg}}} d r \int_{0}^{\beta} d t \int d \phi^{\prime}(-4 r) \\
& =\frac{\beta}{8 G}\left(R_{\mathrm{reg}}^{2}-R_{+}^{2}\right) .
\end{aligned}
$$

This is the same result as the $T=0$ case.

Next, we evaluate the brane action. In three dimensions, the extrinsic curvature satisfies $K=2 T$. For the brane anchored at $\phi^{\prime}=0$, the induced metric reads,

$$
\begin{aligned}
h_{r r}= & \frac{r^{4} T^{2}\left(R_{+}^{2}+R_{-}^{2}\right)}{R_{+}^{2}\left(r^{2}+R_{-}^{2}\right)^{2}\left(T^{2} R_{+}^{2}+R_{-}^{2}-r^{2}\left(T^{2}-1\right)\right)} \\
& +\frac{r^{2}}{\left(r^{2}-R_{+}^{2}\right)\left(r^{2}+R_{-}^{2}\right)}, \\
h_{t r} & =\frac{R_{-} \operatorname{Tr}\left(R_{+}^{2}-r^{2}\right) \sqrt{R_{+}^{2}+R_{-}^{2}}}{R_{+}^{2}\left(r^{2}+R_{-}^{2}\right) \sqrt{r^{2}\left(1-T^{2}\right)+R_{+}^{2} T^{2}+R_{-}^{2}}}, \\
h_{t t}= & \frac{\left(r^{2}-R_{+}^{2}\right)\left(r^{2}+R_{-}^{2}\right)}{r^{2}}+\left(\frac{R_{-}}{R_{+}}-\frac{R_{+} R_{-}}{r^{2}}\right)^{2} r^{2} .
\end{aligned}
$$

From this we find

$$
\operatorname{det}\left(h_{a b}\right)=\frac{r^{2}\left(R_{+}^{2}+R_{-}^{2}\right)}{R_{+}^{2}\left(r^{2}\left(1-T^{2}\right)+R_{+}^{2} T^{2}+R_{-}^{2}\right)} .
$$

On the other hand, in the case where the brane is anchored at $\phi^{\prime}=\pi$, we can repeat the same thing, and the result is the same as above. The brane action can be written as
$S_{\text {brane }}=\frac{T}{8 \pi G} \int_{R_{+}}^{R_{\mathrm{reg}}} d r \int_{0}^{\beta} d t \sqrt{\frac{r^{2}\left(R_{+}^{2}+R_{-}^{2}\right)}{R_{+}^{2}\left(r^{2}\left(1-T^{2}\right)+R_{+}^{2} T^{2}+R_{-}^{2}\right)}}$.

However, the brane contribution for $\phi^{\prime}=0$ and $\phi^{\prime}=\pi$ is canceled as we note in the calculation of the EinsteinHilbert action; the brane is curved in the opposite direction with respect to the bulk space. For the other terms on the conformal boundary, we have just the same term as in the $T=0$ case because in $r \rightarrow \infty$ limit $\phi^{\prime}$ goes to 0 and $\pi$.

Combining them, we obtain

$$
\begin{aligned}
S= & \frac{\beta}{8 G}\left(R_{\mathrm{reg}}^{2}-R_{+}^{2}\right)-\frac{\beta}{8 G}\left(2 R_{\mathrm{reg}}^{2}-R_{+}^{2}+R_{-}^{2}\right) \\
& +\frac{k \beta}{8 G} \sqrt{\left(R_{\mathrm{reg}}^{2}-R_{+}^{2}\right)\left(R_{\mathrm{reg}}^{2}+R_{-}^{2}\right)} \\
\rightarrow & -\frac{\beta\left(R_{+}^{2}+R_{-}^{2}\right)}{16 G},
\end{aligned}
$$

where we take $R_{\text {reg }} \rightarrow \infty$.

If we assume the tensions of the two branes take different values, $T_{0}$ and $T_{\pi}$, respectively, we obtain a nontrivial result. For the Einstein-Hilbert action part, the integral becomes

$$
\begin{aligned}
S_{\mathrm{EH}}= & -\frac{1}{16 G} \int_{R_{+}}^{R_{\mathrm{reg}}} d r \int_{0}^{\beta} d t(-4 r) \\
& \cdot \log \left[\frac{\left(\frac{T_{\pi}}{\sqrt{1-T_{\pi}^{2}}} \sqrt{\frac{R_{+}^{2}+R_{-}^{2}}{r^{2}+R_{-}^{2}}}+\sqrt{\frac{T_{\pi}^{2}}{1-T_{\pi}^{2}}\left(\frac{R_{+}^{2}+R_{-}^{2}}{r^{2}+R_{-}^{2}}\right)+1}\right)}{\left.\left(\frac{T_{0}}{\sqrt{1-T_{0}^{2}}} \sqrt{\frac{R_{+}^{2}+R_{-}^{2}}{r^{2}+R_{-}^{2}}}+\sqrt{\frac{T_{0}^{2}}{1-T_{0}^{2}}\left(\frac{R_{+}^{2}+R_{-}^{2}}{r^{2}+R_{-}^{2}}\right)+1}\right)\right]} .\right.
\end{aligned}
$$

After performing the integral, we finally obtain

$$
\begin{aligned}
S= & -\frac{\beta\left(R_{+}^{2}+R_{-}^{2}\right)}{16 G} \\
& -\frac{\beta\left(R_{+}^{2}+R_{-}^{2}\right)}{16 \pi G R_{+}}\left(\log \left(\frac{1+T_{\pi}}{1-T_{\pi}}\right)-\log \left(\frac{1+T_{0}}{1-T_{0}}\right)\right),
\end{aligned}
$$

where we omit the divergent term, which is proportional to $R_{\text {reg. }}$. For the nonrotating BTZ, this result matches with that in [6].

\section{A ONE-LOOP PARTITION FUNCTION IN THE THERMAL AdS WITH A TENSIONLESS ETW BRANE}

In this section, we will use the explicit form of the heat kernel presented in [3], though our presentation will be brief. Please refer to the original paper if necessary. 


\section{A. A review of the heat kernel method}

The heat kernel method is a convenient way of calculating a one-loop partition function. We follow the analysis in $[3,19-22]$. Let us consider calculating the partition function of a free scalar $\phi$,

$$
Z=\int D \phi e^{-S(\phi)}
$$

The action of the scalar field can be rewritten as

$$
S(\phi)=\int_{M} d^{3} x \sqrt{g} \phi \Delta \phi,
$$

where we omit indices of tensorial structure. Since we are considering a compact space, $\Delta$ has a discrete set of eigenvalues $\lambda_{n}$. The one-loop partition function is expressed as follows:

$$
S^{(1)}=-\frac{1}{2} \log \operatorname{det}(\Delta)=-\frac{1}{2} \sum_{n} \log \lambda_{n} .
$$

If we consider a noncompact space, then the spectrum becomes continuous, and the one-loop partition function is divergent, which is proportional to the volume. This divergence can be absorbed by the renormalization of the Newton constant.

The heat kernel is defined as

$$
K(t, x, y)=\sum_{n} e^{-\lambda_{n} t} \psi_{n}(x) \psi_{n}(y),
$$

which we usually call a propagator. We can normalize the eigenfunctions as

$$
\begin{aligned}
\sum_{n} \psi_{n}(x) \psi_{n}(y) & =\delta^{3}(x-y), \\
\int_{M} d^{3} x \sum_{n} \sqrt{g} \psi_{n}(x) \psi_{m}(x) & =\delta_{n m} .
\end{aligned}
$$

The trace of the heat kernel is given by

$$
\int_{M} d^{3} x \sum_{n} \sqrt{g} K(t, x, x)=\sum_{n} e^{-\lambda_{n} t} .
$$

Using this we can compute the one-loop partition function as an integral over $t$,

$$
S^{(1)}=-\frac{1}{2} \sum_{n} \log \lambda_{n}=\frac{1}{2} \int_{+0}^{\infty} \frac{d t}{t} \int_{M} d^{3} x \sum_{n} \sqrt{g} K(t, x, x) .
$$

We can show the above equation by differentiating with respect to $\lambda_{n}$. Note that it is an identity up to an infinite constant. The point is that $K$ satisfies the heat conduction equation,

$$
\left(\partial_{t}+\Delta_{x}\right) K(t, x, y)=0,
$$

with a boundary condition at $t=0$,

$$
K(0, x, y)=\delta(x, y) .
$$

\section{B. A one-loop partition function in thermal AdS with ETW brane}

In this section, we apply the calculation in [3] to our ETW brane setup. Now consider Poincaré $\operatorname{AdS}_{3}$, whose metric is given by

$$
d s^{2}=\frac{d y^{2}+d z d \bar{z}}{y^{2}} .
$$

Here an ETW brane is placed at $\Re(z)=0$, and the bulk region is defined as $\Re(z)>0$. We note that the ETW brane is connected in the bulk. Since the AdS space is maximally isometric, the geodesic distance $r\left(x, x^{\prime}\right)$ depends only on the chordal distance $u\left(x, x^{\prime}\right)$,

$$
r\left(x, x^{\prime}\right)=\operatorname{arccosh}\left(1+u\left(x, x^{\prime}\right)\right),
$$

where

$$
u\left(x, x^{\prime}\right)=\frac{\left(y-y^{\prime}\right)^{2}+\left|z-z^{\prime}\right|^{2}}{2 y y^{\prime}} .
$$

A thermal AdS can be obtained from an AdS space using the following identification:

$$
(y, z) \sim\left(|q|^{-1} y, q^{-1} z\right),
$$

where $q=e^{2 \pi i \tau}$ and $\tau=\tau_{1}+i \tau_{2}$. In the nonzero $\tau_{1}$ case, the boundary of the BCFT wraps around the torus for many times, and in this case the region on which BCFT lives is not clear because the region is not surrounded by the boundaries. To cure this problem later we will restrict to the special case $q=\bar{q}$. Now we consider applying the method of images to the heat kernel method. The tensionless ETW brane is inserted at $\Re(z)=0$. Then, if we consider a mirror position, $z$ and $\bar{z}$ are mapped to $-\bar{z}$ and $-z$, respectively. Then, the one-loop partition function becomes

$$
\begin{aligned}
S^{(1)}= & \frac{1}{2} \int_{+0}^{\infty} \frac{d t}{t} \int_{\text {thermal AdS }} d^{3} x \sum_{n} \sqrt{g}\left(K^{\boxplus / \mathbb{Z}}(t, x, x)\right. \\
& \left.+K^{\boxplus / \mathbb{Z}}\left(t, x^{\text {mirror }}, x\right)\right) .
\end{aligned}
$$

In the tensionless case the boundary condition for the metric is given by $K_{a b}=0$. We note that we treat this 
condition as an off-shell boundary condition. This means that we must impose the following boundary condition for the perturbation of the metric:

$$
\partial_{x} h_{i j}=0 .
$$

Since the heat kernel on a thermal AdS can be obtained using the method of images from that of an AdS space, we get

$$
\begin{aligned}
S^{(1)}= & \frac{1}{2} \int_{+0}^{\infty} \frac{d t}{t} \int_{\text {thermal AdS }} d^{3} x \sum_{n} \sqrt{g}\left(K^{\llbracket}\left(t, x, \gamma^{n} x\right)\right. \\
& \left.+K^{\llbracket}\left(t, x^{\text {mirror }}, \gamma^{n} x\right)\right) .
\end{aligned}
$$

For a later convenience, we will use a different coordinate,

$$
y=\rho \sin \theta, \quad z=\rho \cos \theta e^{i \phi},
$$

where $1 \leq \rho \leq e^{2 \pi \tau_{2}}, 0 \leq \theta \leq \frac{\pi}{2}$, and $-\frac{\pi}{2} \leq \phi \leq \frac{\pi}{2}$. In terms of this coordinate the geodesic distances are given by

$$
\begin{aligned}
r\left(x, \gamma^{n} x\right) & =\operatorname{arccosh}\left(\frac{\cosh \beta}{\sin ^{2} \theta}-\frac{\cos \alpha}{\tan ^{2} \theta}\right), \\
r\left(x^{\text {mirror }}, \gamma^{n} x\right) & =\operatorname{arccosh}\left(\frac{\cosh \beta}{\sin ^{2} \theta}+\frac{\cos (2 \phi-\alpha)}{\tan ^{2} \theta}\right),
\end{aligned}
$$

where we defined $\alpha=2 \pi n \tau_{1}$ and $\beta=2 \pi n \tau_{2}$.

\section{A one-loop partition function of a scalar field}

The heat kernel of a scalar field on an $\mathrm{AdS}_{3}$ space is given by

$$
K^{H_{3}}\left(t, r\left(x, x^{\prime}\right)\right)=\frac{e^{-\left(m^{2}+1\right) t-\frac{r^{2}}{4 t}}}{(4 \pi t)^{\frac{3}{2}}} \frac{r}{\sinh (r)} .
$$

Let us consider calculating the ordinary (not the mirror) part. A one-loop determinant can be recast as an integral,

$$
-\log \operatorname{det} \Delta=\operatorname{Vol}\left(H_{3} / \mathbb{Z}\right) \int_{0}^{\infty} \frac{d t}{t} \frac{e^{-\left(m^{2}+1\right) t}}{(4 \pi t)^{\frac{3}{2}}}+\sum_{n \neq 0} \int_{0}^{\infty} \frac{d t}{t} \int \frac{d \rho d \theta d \phi \cos \theta}{\rho \sin ^{3} \theta} \frac{e^{-\left(m^{2}+1\right) t-\frac{r^{2}}{4 t}}}{(4 \pi t)^{\frac{3}{2}}} \frac{r}{\sinh (r)},
$$

where we split into the zero mode part and nonzero mode $n \neq 0$. The first term can be easily regularized,

$$
\int_{0}^{\infty} \frac{d t}{t} \frac{e^{-\left(m^{2}+1\right) t}}{(4 \pi t)^{\frac{3}{2}}}=\frac{\left(m^{2}+1\right)^{\frac{3}{2}}}{8 \pi^{\frac{3}{2}}} \int_{0}^{\infty} d k k^{-\frac{5}{2}} e^{-k}=\frac{\left(m^{2}+1\right)^{\frac{3}{2}}}{6 \pi} .
$$

The second term can be calculated directly. Firstly, we change the variable from $\theta$ to $r$, and we get

$$
\sum_{n \neq 0} \int_{0}^{\infty} \frac{d t}{t} \int \frac{d \rho d \theta d \phi \cos \theta}{\rho \sin ^{3} \theta} \frac{e^{-\left(m^{2}+1\right) t-\frac{r^{2}}{4 t}}}{(4 \pi t)^{\frac{3}{2}}} \frac{r}{\sinh (r)}=\sum_{n \neq 0} \int_{0}^{\infty} \frac{d t}{t} \int \frac{d \rho d r d \phi}{\rho} \frac{e^{-\left(m^{2}+1\right) t-\frac{r^{2}}{4 t}}}{(4 \pi t)^{\frac{3}{2}}} \frac{r}{2(\cosh \beta-\cos \alpha)} .
$$

Integrating over $r, \phi$, and $t$ in order, we can reach the final answer,

$$
\begin{aligned}
\sum_{n \neq 0} \int_{0}^{\infty} \frac{d t}{t} \int \frac{d \rho d r d \phi}{\rho} \frac{e^{-\left(m^{2}+1\right) t-\frac{r^{2}}{4 t}}}{(4 \pi t)^{\frac{3}{2}}} \frac{r}{2(\cosh \beta-\cos \alpha)} & =\sum_{n \neq 0} \frac{\sqrt{\pi} \tau_{2}}{4(\cosh \beta-\cos \alpha)} \int_{0}^{\infty} \frac{d t}{t} \frac{e^{-\left(m^{2}+1\right) t-\frac{\left(2 \pi n \tau_{2}\right)^{2}}{4 t}}}{t^{\frac{3}{2}}} \\
& =\sum_{n \neq 0} \frac{e^{-2 \pi n \tau_{2} \sqrt{m^{2}+1}}}{4 n(\cosh \beta-\cos \alpha)}=\sum_{n=1}^{\infty} \frac{|q|^{n\left(1+\sqrt{m^{2}+1}\right)}}{n\left|1-q^{n}\right|^{2}} .
\end{aligned}
$$

Now let us consider the mirror part. Firstly, we consider the $n=0$ case. The strategy is that we firstly fix $\phi$ and integrate $\theta$ or $r$ and then integrate over other variables. This leads to the divergent result even though we use a regularization by a gamma function,

$$
\int_{0}^{\infty} \frac{d t}{t} \int \frac{d \rho d \theta d \phi \cos \theta}{\rho \sin ^{3} \theta} \frac{e^{-\left(m^{2}+1\right) t-\frac{r^{2}}{4 t}}}{(4 \pi t)^{\frac{3}{2}}} \frac{r}{\sinh (r)}=-\frac{\tau_{2} \sqrt{m^{2}+1}}{2} \int_{-\frac{\pi}{2}}^{\frac{\pi}{2}} \frac{d \phi}{1+\cos (2 \phi)} .
$$

However, this divergence can be eliminated by the renormalization of Newton constant, so we will ignore this term. 
Next consider the $n \neq 0$ case. The trick is almost the same, but the situation is slightly changed. The integral over $\phi$ yields

$$
\begin{aligned}
& \sum_{n \neq 0} \int_{0}^{\infty} d t 2 \pi \tau_{2} \frac{e^{-\left(m^{2}+1\right) t-\frac{\left(2 \pi n \tau_{2}\right)^{2}}{4 t}}}{(4 \pi t)^{\frac{3}{2}}} \int_{-\frac{\pi}{2}}^{\frac{\pi}{2}} \frac{d \phi}{|\cos (2 \phi-\alpha)+\cosh (\beta)|} \\
& =\sum_{n \neq 0} \int_{0}^{\infty} d t 2 \pi \tau_{2} \frac{e^{-\left(m^{2}+1\right) t-\frac{\left(2 \pi n \tau_{2}\right)^{2}}{4 t}}}{(4 \pi t)^{\frac{3}{2}}} \frac{\pi}{\sinh (\beta)} \\
& =\sum_{n=1}^{\infty} \frac{|q|^{n\left(1+\sqrt{m^{2}+1}\right)}}{n\left(1-|q|^{2 n}\right)}
\end{aligned}
$$

\section{A one-loop partition function for a vector field}

In this section we consider the vector field contribution to the partition function. For a later convenience, we define a set of new real coordinates,

$$
\begin{aligned}
x & =\Re(z)=\rho \cos \theta \cos \phi, \\
\eta & =\Im(z)=\rho \cos \theta \sin \phi .
\end{aligned}
$$

The propagator has two indices and is invariant under an exchange of those indices. Thus, we can expand the kernel by the basis of $(1,1)$ symmetric bitensor [25],

$$
K_{\mu \nu^{\prime}}\left(t, x, x^{\prime}\right)=F(t, u) \partial_{\mu} \partial_{\nu^{\prime}} u+\partial_{\mu} \partial_{\nu^{\prime}} S(t, u),
$$

where

$$
\begin{aligned}
& F(t, r)=-\frac{e^{-\frac{r^{2}}{4 t}}}{(4 \pi t)^{\frac{3}{2}}} \frac{r}{\sinh r} \\
& S(t, r)=\frac{4}{(4 \pi)^{\frac{3}{2}}} \frac{e^{-\frac{r^{2}}{4 t}} \sinh r}{t} \sqrt{t} \int_{0}^{1} d \xi e^{-t(1-\xi)^{2}} \sinh (r \xi) .
\end{aligned}
$$

The next step is to calculate the derivatives of $u$. In the Poincaré coordinate it can be explicitly given by

$$
\begin{aligned}
\partial_{\mu} \partial_{\nu^{\prime}} u= & -\frac{1}{z_{0} w_{0}}\left\{\delta_{\mu \nu^{\prime}}+\frac{(z-w)_{\mu}}{w_{0}} \delta_{\nu^{\prime} 0}\right. \\
& \left.+\frac{(w-z)_{\nu^{\prime}}}{z_{0}} \delta_{\mu 0}-u \delta_{\mu 0} \delta_{\nu^{\prime} 0}\right\} .
\end{aligned}
$$

Here we note that $z=x$ or $z=x^{\text {mirror }}$, and $w=\gamma^{n} x$. We will present a detailed calculation of these derivatives in Appendix A. Let us consider the ordinary (not the mirror image) contribution. Using those results, we can compute the kernel,

$$
\begin{aligned}
& \int_{0}^{\infty} \frac{d t}{t} \int d^{3} x \sqrt{g} \sum_{n} g^{\mu \rho} \frac{\partial\left(\gamma^{n} x\right)^{\nu^{\prime}}}{\partial x^{\rho}} K_{\mu \nu^{\prime}}^{\mathbb{H}}\left(t, r\left(x, \gamma^{n} x\right)\right) \\
& =\int_{0}^{\infty} \frac{d t}{t} \int d^{3} x \sqrt{g} \sum_{n}\left\{\frac { d ^ { 2 } } { S } d u ^ { 2 } \left\{\left(e^{\beta}-\cosh r\right)\left(e^{-\beta} \cosh r\right)\right.\right. \\
& \quad-2 \cos \alpha(\cosh r-\cosh \beta)\} \\
& \left.\quad+\left(F+\left(\frac{d S}{d u}\right)\right)(\cosh r-2 \cosh \beta-2 \cos \alpha)\right\} \\
& =\operatorname{Vol}(\mathbb{H} / \mathbb{Z}) \int_{0}^{\infty} \frac{d t}{t} \frac{\left(e^{-t}+2+4 t\right)}{(4 \pi t)^{\frac{3}{2}}} \\
& \quad+\sum_{n=0}^{\infty} \int_{0}^{\infty} \frac{d t}{t} \frac{2 \pi^{2} \tau_{2}}{(\cosh \beta-\cos \alpha)} \frac{e^{-\frac{\beta^{2}}{4 t}}}{4 \pi^{\frac{3}{2}} \sqrt{t}}\left(2 \cos \alpha+e^{-t}\right) .
\end{aligned}
$$

The first term can be regularized by considering a massless limit of a massive field,

$$
\begin{aligned}
& \operatorname{Vol}(\mathbb{H} / \mathbb{Z}) \int_{0}^{\infty} \frac{d t}{t} \frac{\left(e^{-t}+2+4 t\right)}{(4 \pi t)^{\frac{3}{2}}} \\
& =\lim _{m \rightarrow 0} \operatorname{Vol}(\mathbb{H} / \mathbb{Z}) \int_{0}^{\infty} \frac{d t}{t} \frac{e^{-m^{2} t}\left(e^{-t}+2+4 t\right)}{(4 \pi t)^{\frac{3}{2}}} \\
& =\operatorname{Vol}(\mathbb{M} / \mathbb{Z}) \frac{1}{(4 \pi)^{\frac{3}{2}}}\left(\frac{4 \sqrt{\pi}}{3}-8 \sqrt{\pi}\right)=-\frac{5}{6 \pi} \operatorname{Vol}(\mathbb{W} / \mathbb{Z}) \text {. }
\end{aligned}
$$

The second term can be calculated straightforwardly,

$$
\begin{aligned}
& \sum_{n=1}^{\infty} \int_{0}^{\infty} \frac{d t}{t} \frac{2 \pi^{2} \tau_{2}}{(\cosh \beta-\cos \alpha)} \frac{e^{-\frac{\beta^{2}}{4 t}}}{4 \pi^{\frac{3}{2}} \sqrt{t}}\left(2 \cos \alpha+e^{-t}\right) \\
& \quad=\sum_{n=1}^{\infty} \frac{2 \cos \alpha+e^{-\beta}}{2 n(\cosh \beta-\cos \alpha)} \\
& \quad=\sum_{n=1}^{\infty} \frac{q^{n}+\bar{q}^{n}+|q|^{2 n}}{n\left|1-q^{n}\right|^{2}} .
\end{aligned}
$$

If we omit the term, which is proportional to the volume, then the answer is just a half of the original result as we expected.

Next, we consider the mirror image part. Since the brane is inserted at $x=0$, we have $x_{\text {mirror }}=-x, \eta_{\text {mirror }}=\eta$, and $\mathrm{y}_{\text {mirror }}=y$. The derivatives of $u$ are also slightly modified, and we present the results in Appendix A. Computations analogous to the previous ones lead to 


$$
\begin{aligned}
& \int_{0}^{\infty} \frac{d t}{t} \int d^{3} x \sqrt{g} \sum_{n} g^{\mu \rho} \frac{\partial\left(\gamma^{n} x\right)^{\nu^{\prime}}}{\partial x^{\rho}} K_{\mu \nu^{\prime}}^{\mathrm{H}}\left(t, r\left(x_{\text {mirror }}, \gamma^{n} x\right)\right) \\
& =\int_{0}^{\infty} \frac{d t}{t} \int d^{3} x \sqrt{g} \sum_{n}\left[\left(\frac{d^{2} S}{d u^{2}}\right)\left\{\left(e^{\beta}-\cosh r\right)\left(e^{-\beta}-\cosh r\right)-2 \cos \alpha(\cosh r-\cosh \beta)\right\}\right. \\
& \left.+\left(F+\left(\frac{d S}{d u}\right)\right)(\cosh r-2 \cosh \beta-2 \cos \alpha)\right] .
\end{aligned}
$$

The above form is the same as the previous result, except that we have different geodesic distances,

$$
r=\operatorname{arccosh}\left(\frac{\cosh \beta}{\sin ^{2} \theta}+\frac{\cos (2 \phi-\alpha)}{\tan ^{2} \theta}\right)
$$

This distance $r$ depends not only $\theta$, but also $\phi$. To proceed, we fix $\phi$ and integrate $\theta$ (or $r$ ) firstly. The integral over $r$ gives the same as the original calculation because it is independent of $\phi$,

$$
\begin{aligned}
& \int_{0}^{\infty} \frac{d t}{t} \int d^{3} x \sqrt{g} \sum_{n}\left[\left(\frac{d^{2} S}{d u^{2}}\right)\left\{\left(e^{\beta}-\cosh r\right)\left(e^{-\beta}-\cosh r\right)-2 \cos \alpha(\cosh r-\cosh \beta)\right\}\right. \\
& \left.+\left(F+\left(\frac{d S}{d u}\right)\right)(\cosh r-2 \cosh \beta-2 \cos \alpha)\right] \\
& =\int_{0}^{\infty} \frac{d t}{t} \sum_{n} 2 \pi \tau_{2} \int_{-\frac{\pi}{2}}^{\frac{\pi}{2}} d \phi\left[\frac{1}{2(\cosh \beta+\cos (2 \phi-\alpha))} \frac{e^{-\frac{\beta^{2}}{4 t}}}{4 \pi^{\frac{3}{2}} \sqrt{t}}\left(2 \cos \alpha+e^{-t}\right)\right] .
\end{aligned}
$$

The integral over $\phi$ can also be evaluated,

$$
\int_{-\frac{\pi}{2}}^{\frac{\pi}{2}} d \phi \frac{1}{(\cosh \beta+\cos (2 \phi-\alpha))}=\frac{\pi}{\sinh \beta}
$$

The mirror image contribution finally becomes

$$
\int_{0}^{\infty} \frac{d t}{t} \int d^{3} x \sqrt{g} \sum_{n} g^{\mu \rho} \frac{\partial\left(\gamma^{n} x\right)^{\nu^{\prime}}}{\partial x^{\rho}} K_{\mu \nu^{\prime}}^{\boxplus n}\left(t, r\left(x_{\text {mirror }}, \gamma^{n} x\right)\right)=\sum_{n=1}^{\infty} \frac{2 \cos \alpha+e^{-\beta}}{2 n \sinh \beta}=\sum_{n=1}^{\infty} \frac{q^{n}+\bar{q}^{n}+|q|^{2 n}}{n\left(1-|q|^{2 n}\right)} .
$$

\section{E. A one-loop partition function of a symmetric spin-2 field}

In the calculation of the vector field one-loop partition function we see that the trace of the kernel in the mirror part is the same as that in the ordinary part. This seems to be somewhat miraculous at a first sight. However, this is not surprising because the trace of the kernel can only depend on geodesic distance $r$.

The kernel of the symmetric spin-2 field can be expanded by the basis of $(2,2)$ symmetric tensors $[3,25]$. Since they are complicated, we do not want to write explicitly here.

The one-loop determinant of a symmetric traceless tensor is given by

$$
-\log \operatorname{det} \Delta=\int_{0}^{\infty} \frac{d t}{t} \int d^{3} x \sqrt{g} \sum_{n}\left\{g^{\mu \rho} \frac{\partial\left(\gamma^{n} x\right)^{\mu^{\prime}}}{\partial x^{\rho}} g^{\nu \sigma} \frac{\partial\left(\gamma^{n} x\right)^{\nu^{\prime}}}{\partial x^{\sigma}}\left(K_{\mu \mu^{\prime}, \nu \nu^{\prime}}^{\boxplus}\left(t, r\left(x, \gamma^{n} x\right)\right)+K_{\mu \mu^{\prime}, \nu \nu^{\prime}}^{\boxplus}\left(t, r\left(x_{\text {mirror }}, \gamma^{n} x\right)\right)\right)\right\} .
$$

Let us consider the calculation of the first term. The integral over $r$ gives 


$$
\begin{aligned}
& \int_{0}^{\infty} \frac{d t}{t} \int d^{3} x \sqrt{g} \sum_{n} g^{\mu \rho} \frac{\partial\left(\gamma^{n} x\right)^{\mu^{\prime}}}{\partial x^{\rho}} g^{\nu \sigma} \frac{\partial\left(\gamma^{n} x\right)^{\nu^{\prime}}}{\partial x^{\sigma}} K_{\mu \mu^{\prime}, \nu \nu^{\prime}}^{\llbracket \Perp}\left(t, r\left(x, \gamma^{n} x\right)\right) \\
& =\sum \int_{0}^{\infty} \frac{d t}{t} \frac{2 \pi^{2} \tau_{2}}{2(\cosh \beta-\cos \alpha)} \frac{e^{-\frac{\beta^{2}}{4 t}}}{2 \pi^{\frac{3}{2}} \sqrt{t}}\left(e^{-t} \cos 2 \alpha+e^{-4 t} \cos \alpha+\frac{e^{-5 t}}{2}\right) \\
& =\sum_{n=1}^{\infty} \frac{1}{n(\cosh \beta-\cos \alpha)}\left(e^{-\beta} \cos 2 \alpha+e^{-2 \beta} \cos \alpha+\frac{e^{-\sqrt{5} \beta}}{2}\right),
\end{aligned}
$$

where we omit an $n=0$ term because it is proportional to the volume. Next, we consider the second term. We can perform the integration similarly,

$$
\begin{aligned}
\int_{0}^{\infty} & \frac{d t}{t} \int d^{3} x \sqrt{g} \sum_{n}\left\{g^{\mu \rho} \frac{\partial\left(\gamma^{n} x\right)^{\mu^{\prime}}}{\partial x^{\rho}} g^{\nu \sigma} \frac{\partial\left(\gamma^{n} x\right)^{\nu^{\prime}}}{\partial x^{\sigma}} K_{\mu \mu^{\prime}, \nu \nu^{\prime}}^{\mathbb{1}}\left(t, r\left(x_{\text {mirror }}, \gamma^{n} x\right)\right)\right\} \\
= & \sum_{n} \int_{0}^{\infty} \frac{d t}{t} 2 \pi \tau_{2} \int_{-\frac{\pi}{2}}^{\frac{\pi}{2}} d \phi\left\{\frac{1}{2(\cosh \beta+\cos (2 \phi-\alpha))} \frac{e^{-\frac{\beta^{2}}{4 t}}}{2 \pi^{\frac{3}{2}} \sqrt{t}}\left(e^{-t} \cos 2 \alpha+e^{-4 t} \cos \alpha+\frac{e^{-5 t}}{2}\right)\right\} \\
= & \int_{0}^{\infty} \frac{d t}{t} 2 \pi \tau_{2} \int_{-\frac{\pi}{2}}^{\frac{\pi}{2}} d \phi \frac{1}{2(1+\cos (2 \phi))} \frac{1}{2 \pi^{\frac{3}{2}} \sqrt{t}}\left(e^{-t}+e^{-4 t}+\frac{e^{-5 t}}{2}\right) \\
& +\sum_{n} \int_{0}^{\infty} \frac{d t}{t} 2 \pi \tau_{2} \frac{\pi}{\sinh \beta} \frac{e^{-\frac{\beta^{2}}{4 t}}}{2 \pi^{\frac{3}{2}} \sqrt{t}}\left(e^{-t} \cos 2 \alpha+e^{-4 t} \cos \alpha+\frac{e^{-5 t}}{2}\right)
\end{aligned}
$$

The first term contains a divergent integral, and here we will ignore it. The second term can be simplified as follows:

$\sum_{n} \int_{0}^{\infty} \frac{d t}{t} 2 \pi \tau_{2} \frac{\pi}{\sinh \beta} \frac{e^{-\frac{\beta^{2}}{4 t}}}{2 \pi^{\frac{3}{2}} \sqrt{t}}\left(e^{-t} \cos 2 \alpha+e^{-4 t} \cos \alpha+\frac{e^{-5 t}}{2}\right)=\sum_{n=1}^{\infty} \frac{1}{n \sinh \beta}\left(e^{-\beta} \cos 2 \alpha+e^{-2 \beta} \cos \alpha+\frac{e^{-\sqrt{5} \beta}}{2}\right)$

\section{F. A one-loop partition function for gravity}

We will consider a linearized graviton perturbation $h_{\mu \nu}$ around the AdS background $g_{\mu \nu}$. The Einstein-Hilbert action in the three dimensions with a negative cosmological constant is given by

$$
S_{G R}=-\frac{1}{16 \pi G} \int d^{3} x(R+2) \sqrt{g}
$$

We will use the gauge of [1], where we add the gauge-fixing term to (75),

$S_{G F}=\frac{1}{32 \pi G} \int d^{3} x \sqrt{g} \nabla^{\mu}\left(h_{\mu \sigma}-\frac{1}{2} g_{\mu \sigma} h\right) \nabla^{\nu}\left(h_{\nu}^{\sigma}-\frac{1}{2} \delta_{\nu}^{\sigma} h\right)$

It is convenient to define the traceless part and the trace part,

$$
\begin{aligned}
\phi_{\mu \nu} & =h_{\mu \nu}-\frac{1}{3} g_{\mu \nu} h_{\rho}^{\rho} \\
\phi & =h_{\rho}^{\rho} .
\end{aligned}
$$

The gauge-fixed action is given by [3]

$$
\begin{aligned}
S= & -\frac{1}{32 \pi G} \int d^{3} x \sqrt{g}\left\{\frac{1}{2} \phi_{\mu \nu}\left(g^{\mu \rho} g^{\nu \sigma} \nabla^{2}+2 R^{\mu \rho \nu \sigma}\right) \phi_{\rho \sigma}\right. \\
& \left.-\frac{1}{12} \phi\left(\nabla^{2}-4\right) \phi\right\} .
\end{aligned}
$$

We will wick-rotate $\phi \rightarrow i \phi$ in order to make the kinetic term positive definite. The gauge-fixing term introduces a Fadeev-Popov field, which is a Grassmann-odd vector field,

$$
S_{\text {ghost }}=\frac{1}{32 \pi G} \int d^{3} x \sqrt{g} \overline{\eta_{\mu}}\left(-g^{\mu \nu} \nabla^{2}-R^{\mu \nu}\right) \eta^{\nu} .
$$


Therefore, the gravity partition function can be obtained by subtracting the contribution of the vector ghost field with $m^{2}=4$ and the scalar field with $m^{2}=4$, which corresponds to the trace part of the fluctuation,

$$
\begin{aligned}
\log Z_{\text {gravity }}^{1-\text { loop }} \\
=-\frac{1}{2} \log \operatorname{det} \Delta^{\text {graviton }}+\log \operatorname{det} \Delta^{\text {vector }}-\frac{1}{2} \log \operatorname{det} \Delta^{\text {scalar }} \\
=\sum_{n=2}^{\infty}-\frac{\log \left|1-q^{n}\right|^{2}}{2} \\
\quad+\sum_{n=1}^{\infty} \frac{1}{2 n} \frac{1}{1-|q|^{2 n}}\left(q^{2 n}\left(1-\bar{q}^{n}\right)+\bar{q}^{2 n}\left(1-q^{n}\right)\right) .
\end{aligned}
$$

The first term is just a half of the contribution in the original theory, and the second term comes from the mirror contribution.

\section{A PHYSICAL INTERPRETATION}

\section{A. A BCFT interpretation of the partition function}

In this section we summarize the results of the partition functions in the previous section and give a physical interpretation from the BCFT viewpoint. Before we move on, let us review our strategy of the calculation. We calculated the partition function using the method of images. In our calculation we approximate the ETW brane as the hard wall in the bulk geometry, which means that the location of the ETW brane is determined by solving the Neumann boundary condition for the tensionless ETW brane $K_{a b}=0$. Then, we apply the method of images to the background AdS metric and evaluate the fluctuation of the metric around the solution. Because the brane is tensionless and the action is proportional to the tension, here we obtain the contributions from the ETW brane only through the method of images.

Let us summarize our result. We firstly consider the scalar part. The partition function of the free scalar field with Neumann boundary condition on the ETW brane is given by

$$
\begin{aligned}
Z_{\text {Scalar }}= & \left(\prod_{l=0}^{\infty} \prod_{l^{\prime}=0}^{\infty} \frac{1}{\sqrt{1-q^{l+h} \bar{q}^{l^{\prime}+h}}}\right) \\
& \cdot\left(\prod_{m=0}^{\infty} \frac{1}{\sqrt{1-q^{m+h} \bar{q}^{m+h}}}\right) .
\end{aligned}
$$

The first term describes the ordinary contribution and the second one does the mirror image one. The former has a clear interpretation: it comes from a primary field with conformal dimension $h$ and a summation over its descendants. The square root is present because we take the volume of the space to be a half of the original AdS space. The second term is coming from the mirror image effect of the original field. However, because in the AdS/BCFT case, the rotational symmetry of the torus is broken due to the ETW brane, we have only one real parameter $\beta$. Therefore, we expect that we should set $q=\bar{q}$ physically. In this case the expression gets simplified,

$$
\begin{aligned}
Z_{\text {Scalar }}= & \left(\prod_{l=0}^{\infty} \prod_{l^{\prime}=0}^{\infty} \frac{1}{\sqrt{1-q^{l+l^{\prime}+2 h}}}\right) \\
& \cdot\left(\prod_{m=0}^{\infty} \frac{1}{\sqrt{1-q^{2(m+h)}}}\right) .
\end{aligned}
$$

Next, the vector field partition function is expressed as follows:

$$
\begin{aligned}
Z_{\mathrm{vector}}= & \left(\prod_{l, l^{\prime}=0}^{\infty} \frac{1}{\sqrt{1-q^{l+1} \bar{q}^{l^{\prime}}} \sqrt{1-q^{l} \bar{q}^{l^{\prime}+1}}} \frac{1}{\sqrt{1-q^{l+h} \bar{q}^{l^{\prime}+h}}}\right) \\
& \cdot\left(\prod_{l=0}^{\infty} \frac{1}{\sqrt{1-q^{l+1} \bar{q}^{l}} \sqrt{1-q^{l} \bar{q}^{l+1}}} \frac{1}{\sqrt{1-q^{l+h} \bar{q}^{l+h}}}\right),
\end{aligned}
$$

as is the case before the first term describes the ordinary contribution and the second one does the mirror effect. In both lines, the second factor is due to the contribution from the longitudinal scalar mode. The rest is coming from the transverse vector mode. The summation is over $L_{-1}$ and $\bar{L}_{-1}$ plus descendants contribution, which represents a massless spin-1 particle. For a massive vector field, we can replace the powers of $q$, i.e., $1+l$ with $1+l+h$. By setting $q=\bar{q}$ as before for the consistent profile of ETW brane, the partition function takes the simplified form,

$$
\begin{aligned}
Z_{\mathrm{vector}}= & \left(\prod_{l, l^{\prime}=0}^{\infty} \frac{1}{1-q^{l+l^{\prime}+1}} \frac{1}{\sqrt{1-q^{l+l^{\prime}+2 h}}}\right) \\
& \cdot\left(\prod_{l=0}^{\infty} \frac{1}{1-q^{2 l+1}} \frac{1}{\sqrt{1-q^{2(l+h)}}}\right) .
\end{aligned}
$$

Finally, let us consider the gravity partition function. After some algebras, we reach the expression

$$
\begin{aligned}
Z_{\text {gravity }}= & \left(\prod_{m=2}^{\infty} \frac{1}{\left|1-q^{m}\right|}\right) \\
& \cdot\left(\prod_{l=0}^{\infty} \frac{\sqrt{1-q^{l+2} \bar{q}^{l+1}} \sqrt{1-q^{l+1} \bar{q}^{l+2}}}{\sqrt{1-q^{l+2} \bar{q}^{l}} \sqrt{1-q^{l} \bar{q}^{l+2}}}\right) .
\end{aligned}
$$

The first part gives the summation over vacuum and its chiral Virasoro descendants. The second term looks complicated, but it has important physical meaning: the numerator represents the massive vector field with $h=1$, and the denominator represents a massless spin-2 field. In $\mathrm{AdS}_{3} / \mathrm{CFT}_{2}$, the bulk field with a spin $l$ and a mass $M$ is dual to an operator with a conformal dimension $2 h$, 


$$
\begin{aligned}
M^{2} & =\Delta(\Delta-2)+l^{2} \\
\Delta & =2 h+l .
\end{aligned}
$$

Therefore, the $h=1$ massive vector field has a mass $M^{2}=4$ in the bulk, which exactly matches what appears as a ghost vector field when we fix the gauge redundancy of gravity. We will revisit this point in Sec. IV C.

Finally, by setting $q=\bar{q}$ again for a consistent profile of the ETW brane, we obtain

$$
Z_{\text {gravity }}=\prod_{l=0}^{\infty} \frac{1}{\left(1-q^{2 l+2}\right)^{2}} .
$$

This has a peculiar exponent $2 l+2$ when compared with the original result (1) without ETW branes [3]. We note that even though taking into account the ghost contribution, this partition function seems to be physical in a sense that the coefficients of the expansion in powers of $q$, i.e., the numbers of states at that conformal dimension, are nonnegative. Interestingly, the ghost contribution goes away.

\section{B. A one-loop partition function with the Dirichlet boundary condition}

So far we impose the Neumann boundary condition on the ETW brane, following the standard prescription of AdS/BCFT. However, it is also interesting to consider more general boundary conditions. Therefore, in this section we repeat the similar calculation now for the Dirichlet boundary condition on the ETW brane. Refer to [12] which suggests a possibility of using the Dirichlet boundary condition in related holographic computations. In this section we only present the results briefly, leaving the details in the Appendix B.

The procedure to find results in the Dirichlet case is very simple: just flip the sign of the mirror image contribution. This leads to

$$
\begin{aligned}
& Z_{\text {Scalar }}=\frac{\left(\prod_{m=0}^{\infty} \frac{1}{\sqrt{1-q^{m+h} \bar{q}^{m+h}}}\right)}{\left(\prod_{l, l^{\prime}=0}^{\infty} \frac{1}{\sqrt{1-q^{l+h} \bar{q}^{l^{\prime}+h}}}\right)}, \\
& Z_{\mathrm{vector}}=\frac{\prod_{l=0}^{\infty} \frac{1}{\sqrt{1-q^{l+1} \bar{q}^{l}} \sqrt{1-q^{l} \bar{q}^{l+1}}} \frac{1}{\sqrt{1-q^{l+h} \bar{q}^{l+h}}}}{\prod_{l, l^{\prime}=0}^{\infty} \frac{1}{\sqrt{1-q^{l+1} \bar{q}^{I^{\prime}}} \sqrt{1-q^{l} \bar{q}^{l^{\prime}+1}}} \frac{1}{\sqrt{1-q^{l+h} \bar{q}^{I^{\prime}+h}}}}, \\
& Z_{\text {gravity }}=\left(\prod_{m=2}^{\infty} \frac{1}{\left|1-q^{m}\right|}\right) \\
& \cdot\left(\prod_{l=0}^{\infty} \frac{\sqrt{1-q^{l+2} \bar{q}^{l}} \sqrt{1-q^{l} \bar{q}^{l+2}}}{\sqrt{1-q^{l+2} \bar{q}^{l+1}} \sqrt{1-q^{l+1} \bar{q}^{l+2}}}\right) .
\end{aligned}
$$

When we set $q=\bar{q}$ to have a consistent profile of ETW branes, we obtain

$$
\begin{gathered}
Z_{\text {Scalar }}=\prod_{l, l^{\prime}=0}^{\infty} \frac{1}{\sqrt{1-q^{l+l^{\prime}+2 h}}}, \\
Z_{\text {vector }}^{\text {transverse }}=\prod_{l, l^{\prime}=0}^{\infty} \frac{1}{1-q^{l+l^{\prime}+1}}, \\
Z_{\text {gravity }}=\prod_{l=0}^{\infty} \frac{1}{\left(1-q^{2 l+3}\right)^{2}},
\end{gathered}
$$

where we omit the longitudinal modes in the vector field partition function. The multiples of nondiagonal contributions lead to the gravity partition function, which includes only the odd integer modes unlike the Neumann case.

\section{A consistency of the boundary condition}

In this section we will be more careful about the boundary conditions. Refer to $[10,11,22,26]$ for a list of early works. Usually, in gravity we impose the Neumann or the Dirichlet boundary condition (B.C.) on a boundary. At the tree level, these conditions harm nothing in calculating physical quantities, such as the partition function. However, the situation changes when we consider quantities at the one-loop level. At the linearized level of the metric, we need a gauge-fixing term and a ghost field. The boundary conditions for these fields affect the one-loop partition function and, what is more, the gauge transformation for the metric may not be compatible with a given boundary condition. Thus, the boundary operator $B$ defines a consistent gauge-invariant boundary condition,

$$
\left.B \phi\right|_{\partial M}=0,
$$

if and only if there exists boundary conditions for the corresponding gauge parameter $\xi$,

$$
B_{\xi} \xi=0,
$$

such that

$$
B \delta_{\xi} \phi=0
$$

This condition ensures the validity of the one-loop calculation of the gauge invariance with the Faddeev-Popov trick. Now we revisit our problem. In our calculation we impose the Neumann or the Dirichlet conditions for all fields, including ghost fields. Let us check if this is reasonable or not. Firstly, we consider the Neumann case. Note that we now split the metric into the background AdS Poincaré metric $g_{\mu \nu}$ and the metric fluctuation $h_{\mu \nu}$. We impose the Neumann B.C. on $h_{i j}$,

$$
\left.\partial_{x} h_{i j}\right|_{x=0}=0,
$$


where $i, j$ represents the tangential direction along the brane. This comes from the original condition $K_{a b}=0$. The symmetric traceless tensor $\phi_{\mu \nu}$ and trace part $\phi$ are given by

$$
\begin{aligned}
\phi_{\mu \nu} & =h_{\mu \nu}-\frac{1}{3} g_{\mu \nu} \phi, \\
\phi & =h_{\rho}^{\rho} .
\end{aligned}
$$

Therefore, we should also impose the Neumann B.C. for these fields,

$$
\begin{aligned}
\left.\partial_{x} \phi_{i j}\right|_{x=0} & =0, \\
\left.\partial_{x} \phi\right|_{x=0} & =0 .
\end{aligned}
$$

To see the condition for the ghost field, we remember that it generates a gauge transformation for the metric,

$$
h_{\mu \nu} \rightarrow h_{\mu \nu}+\nabla_{\mu} \eta_{\nu}+\nabla_{\nu} \eta_{\mu} .
$$

Let us check the gauge invariance of the Neumann B.C. The condition for the ghost field is

$$
\partial_{x}\left(\nabla_{i} \eta_{j}+\nabla_{j} \eta_{i}\right)=0 .
$$

Since the Christoffel symbols are functions of $y$, we can satisfy this equation if we impose the Neumann B.C. for the ghost vector field $\eta_{i}$ and the antighost vector field $\overline{\eta_{i}}$.

Next, we move to the components in the normal direction. If we allow the boundary to fluctuate infinitesimally along the $x$ direction, then we should impose the Neumann boundary condition for $\eta_{x}$ and $\overline{\eta_{x}}$,

$$
\begin{aligned}
& \left.\partial_{x} \eta_{x}\right|_{x=0}=0, \\
& \left.\partial_{x} \bar{\eta}_{x}\right|_{x=0}=0 .
\end{aligned}
$$

Additionally, the BRST variation for $\overline{\eta_{\mu}}$ is given by

$$
\delta \overline{\eta_{\mu}}=\nabla^{\nu} h_{\mu \nu}-\frac{1}{2} \partial_{\mu} \phi
$$

This variation should also satisfy (102). Thus, we can get the boundary condition of the metric along the normal direction,

$$
\left.\partial_{x} \nabla_{\nu} h^{\mu \nu}\right|_{x=0}=0 .
$$

Since the Christoffel symbols are only functions of $y$, we can satisfy the above equation if we impose

$$
\left.\partial_{x} h_{\mu \nu}\right|_{x=0}=0 .
$$

This is what we explicitly assumed in Sec. III. However, we can consider one more possible boundary condition. If we strictly fix the boundary to be on $x=0$, then we should impose the Dirichlet boundary condition for $\eta_{x}$ and $\overline{\eta_{x}}$,

$$
\begin{aligned}
& \left.\eta_{x}\right|_{x=0}=0, \\
& \left.\bar{\eta}_{x}\right|_{x=0}=0 .
\end{aligned}
$$

Correspondingly, the BRST variation of the ghost field changes as

$$
\delta \overline{\eta_{\mu}}=\nabla^{\nu} h_{\mu \nu}-\frac{1}{2} \partial_{\mu} \phi=0,
$$

at the boundary $x=0$. This equation determines the boundary condition for the metric along $x$ direction.

Next, we consider the Dirichlet case. For the tangential direction, we should impose the Dirichlet B.C.,

$$
\begin{aligned}
h_{i j} & =0, \\
\phi_{i j} & =\phi=0, \\
\eta_{i} & =\overline{\eta_{i}}=0,
\end{aligned}
$$

at the boundary $x=0$. If we require that the gauge transformation should vanish at $x=0$, then we get

$$
\begin{aligned}
& \left.\eta_{x}\right|_{x=0}=0, \\
& \left.\bar{\eta}_{x}\right|_{x=0}=0 .
\end{aligned}
$$

The BRST variation gives additional constraints on the metric

$$
\delta \overline{\eta_{\mu}}=\nabla^{\nu} h_{\mu \nu}-\frac{1}{2} \partial_{\mu} \phi=0 .
$$

This result is previously discussed in [26] and is somewhat remarkable; in the Neumann case we show that we can impose the Neumann condition for all fields along the $x$ direction at the boundary. However, in the Dirichlet case we must impose the Neumann-like boundary condition for the metric as in (110). Therefore, our calculation in Sec. III in the Dirichlet case is not BRST invariant because we previously imposed the Dirichlet boundary condition for all the fields and all the components at the boundary. For the completion of classifying the boundary condition, we consider one more case. If we allow the boundary to fluctuate along the $x$ direction, we get

$$
\partial_{x} \eta_{x}=\partial_{x} \overline{\eta_{x}}=0
$$

at the boundary. Then, BRST variation gives 


$$
\partial_{x}\left(\nabla_{\nu} h^{\mu \nu}-\frac{1}{2} \partial^{\nu} \phi\right)=0
$$

at the boundary. However, in the Dirichlet case we should consider one more problem: the ellipticity of the differential operators. In [26], the author discussed that Euclidean linearized gravity with purely the Dirichlet B.C. is not elliptic and hence perturbatively ill defined. We note that in the AdS/CFT case we can allow the Dirichlet B.C. up to the Weyl transformation of the metric, and this B.C. is elliptic [26]. As a consistency check, let us consider the ellipticity of the Neumann B.C. at the level of [26]. Now we impose the Neumann B.C. on all the components of the metric. Then the metric can be written as

$$
h_{\mu \nu}=\zeta_{\mu \nu} \cos \left(k_{x} x\right) \exp \left(i k_{i} x^{i}\right) .
$$

If we differentiate with respect to the $x$, then at the boundary it vanishes automatically.

In the Dirichlet case, we did not notice any pathology in our calculation of the partition function. However, we already know that this boundary condition is not elliptic. Indeed, we can see the breakdown of the ellipticity in a similar way to the above case. We can take the Fourier transformation of the kernel, and $x$ dependence comes from $\exp (i k x)$. Then, the above argument shows that in the Dirichlet case the B.C. is not elliptic. We expect that in our calculation the infinitely many zero modes are hidden in our regularization by the zeta function.

We summarize the above discussion. Firstly, in both the Neumann and the Dirichlet case, we have two sets of boundary conditions for the normal component of the vector fields like in (102) and (106). We found that our calculation in Sec. III will be consistent with BRST invariance for the Neumann boundary condition on all the fields and all the components. On the other hand, if we impose the Dirichlet B.C. on all the components of the metric, then the differential operator is not elliptic, and thus it is perturbatively ill defined. However, in the Neumann case, we can check that the differential operator is elliptic at the level of [26].

\section{D. $S L(2, \mathbb{Z})$ summation of the partition function}

Since we derived the one-loop partition function in Sec. III, here we would like to consider taking $S L(2, \mathbb{Z})$ summation as in $[4,14]$. At first sight this seems to be hard because the first term in (85) cannot be expanded as a polynomial of $q$ and $\bar{q}$. However, physically we remember that we can set $q=\bar{q}$, so we will use (87) and (93).

Firstly, let us consider the Neumann case. In Sec. I we calculated the tree-level partition function of the BTZ black hole. To derive the tree-level partition function of the thermal AdS, we can change the modular parameter as $\tau \rightarrow-\frac{1}{\tau}$. Therefore, the partition function of the thermal AdS is given by

$$
\begin{aligned}
Z_{0,1}(\tau)= & |q \bar{q}|^{-\frac{k}{2}}\left(\prod_{m=2}^{\infty} \frac{1}{\left|1-q^{m}\right|}\right) \\
& \cdot\left(\prod_{l=0}^{\infty} \frac{\sqrt{1-q^{l+2} \bar{q}^{l+1}} \sqrt{1-q^{l+1} \bar{q}^{l+2}}}{\sqrt{1-q^{l+2} \bar{q}^{l}} \sqrt{1-q^{l} \bar{q}^{l+2}}}\right) \\
= & q^{-k} \prod_{l=0}^{\infty} \frac{1}{\left(1-q^{2 l+2}\right)^{2}},
\end{aligned}
$$

where $k=\frac{1}{16 G}$. The whole classical Einstein solution with the boundary torus is obtained by implementing the modular transformation,

$$
\tau \rightarrow \gamma \tau=\frac{a \tau+b}{c \tau+d},
$$

where $\gamma \in S L(2, \mathbb{Z}) /\{ \pm 1\}$. Hence, here we can take $c>0$ and sum over $(c, d)$, which are relatively prime integers. The full partition function can be written as

$$
\begin{aligned}
Z(\tau) & =\sum_{(c, d)} q^{-k} \prod_{l=0}^{\infty} \frac{1}{\left(1-q^{2 l+2}\right)^{2}} \\
& =\sum_{(c, d)} \frac{q^{-k+\frac{1}{6}}}{\eta(2 \tau)^{2}} \\
& =\left.\frac{1}{\sqrt{\Im(2 \tau)} \eta(2 \tau)^{2}} \sum_{(c, d)}\left(\sqrt{\Im(2 \tau)} q^{-k+\frac{1}{6}}\right)\right|_{\gamma} \\
& =\frac{E\left(\tau ; \frac{k}{2}-\frac{1}{12}, 0\right)}{\sqrt{\Im(2 \tau)} \eta(2 \tau)^{2}},
\end{aligned}
$$

where we define $E(\tau ; n, m)=\sum_{(c, d)}\left(\sqrt{\Im(2 \tau)} q^{n} \bar{q}^{m}\right)$. Here we use the modular invariance of $\sqrt{\Im(2 \tau)} \eta(2 \tau)^{2}$.

Next, we consider the Dirichlet case. As in the Neumann case, the partition function becomes

$$
\begin{aligned}
Z(\tau) & =\sum_{(c, d)} q^{-k-\frac{1}{12}} \frac{\eta(2 \tau)^{2}}{\eta(\tau)^{2}}(1-q)^{2} \\
& =\left.\frac{\eta(2 \tau)^{2} \sqrt{\Im(2 \tau)}}{\eta(\tau)^{2} \sqrt{\Im(\tau)}} \sum_{(c, d)}\left(q^{-k-\frac{1}{12}}(1-q)^{2} \frac{\sqrt{\Im(\tau)}}{\sqrt{\Im(2 \tau)}}\right)\right|_{\gamma}
\end{aligned}
$$

Though we can continue the calculation as in [4], we stop here and briefly discuss how to treat this partition function. Firstly, this Poincaré series is divergent, so we need some regularization. One possible way is that we consider the following convergent series:

$$
\sum_{(c, d)}(\Im(2 \tau))^{s} q^{-k+\frac{1}{6}}
$$


This series is convergent for $\Re(s)>1$. However, as is presented in [4], we can take an analytic continuation to $\Re(s) \leq 1$, and especially at $s=\frac{1}{2}$, this series gets regular. We expect that the spectrum has a negative density of states as is the case in pure gravity [27,28]. It will be an interesting future direction to specify the black-hole microstates using the modularity of the theory.

\section{E. One-loop exactness of the partition function}

One-loop exactness of the partition function is an important problem, as in any other theory. Pure gravity in three dimensions is known to be one-loop exact because the bulk diffeomorphism is governed by the Virasoro symmetry, hence the partition function does not suffer any quantum correction other than the Virasoro descendants. We expect the same property in this study, though we have not explicitly shown it. In the two-dimensional BCFT cases, we can use the double trick as explained in [29], which leads to the identification

$$
L_{n}=\overline{L_{-n}} .
$$

Therefore, a half of the Virasoro symmetry or only the chiral mode survives. This also guarantees that if we can properly calculate, respecting the BRST invariance, then the partition function of our case will be one-loop exact.

\section{CONCLUSIONS AND DISCUSSIONS}

In this paper we studied the partition function in the three dimensional AdS/BCFT model with one-loop quantum corrections. First, we calculated the tree-level partition function of the BTZ black hole in the presence of the end of the world brane with arbitrary tension. The result matches with the previous results in the nonrotating case. At the one-loop level, we calculated the thermal AdS partition function with a tensionless brane. We found that the result depends heavily on the choice of sets of boundary conditions. We explicitly calculated the partition function in the case where all the components of all the fields satisfy the Neumann boundary condition and also in another case where all the components of all the fields satisfy the Dirichlet boundary condition. In the Neumann case, we expect that the system will be consistent with the BRST quantization. We found that the partition function actually contains remnants of ghost fields. Note that this ghost mode does not arise from a wrong sign of kinetic terms but does from a fermionic spin-1 field of the BRST ghost. However, if we consider the physically sensible profile of end of the world branes, we need to set $q=\bar{q}$. This leads to a partition function with a healthy spectrum such that the number of states at each energy level is non-negative. This suggests that the AdS/BCFT formulation with the Neumann boundary condition is consistent at the one-loop level.
In the Dirichlet case, on the other hand, we also encounter unphysical modes. There are other possible sets of boundary conditions, namely, mixed boundary conditions for each component. However, in this paper we have not calculated them due to its computational difficulty. This will be one of our future problems. It is also possible that there might be sensible models of the gravity with boundaries by adding a suitable set of matter fields. For example, refer to [11] for an argument using supersymmetry transformations. Therefore, it will be interesting to specify the minimal set of boundary conditions and matter fields to make the theory well defined. For another future direction, it will be important to specify what kind of mode localizes on the brane or decouples from the bulk. As another route to gravity, it may be interesting to understand our problem in the Chern-Simons formulation. We expect that in this setup we can understand the edge modes more clearly because we can only consider the boundary conditions for gauge fields.

In order to derive the full partition function, we should sum over all possible geometries with a given boundary condition [30]. In this work, the bulk geometry is a half of the solid torus, therefore, we can sum over the torus moduli $S L(2, \mathbb{Z})$. As we discussed briefly in Sec. IV, the computation is similar to the one in [4]. It will be an interesting direction to develop this calculation further and analyze the spectrum using modular bootstrap in BCFTs as in the pure gravity case $[27,28]$.

\section{ACKNOWLEDGMENTS}

We thank T. Takayanagi for correcting some errors in manuscripts and for encouragement. We are also grateful to T. Nishioka, T. Ugajin, and D. Vassilevich for valuable comments and discussions.

\section{APPENDIX A: DERIVATIVES OF A GEODESIC DISTANCE}

Here we present the calculation of the derivatives of $u$. Ordinary contributions are given as follows:

$$
\begin{aligned}
\partial_{y} \partial_{x^{\prime}} u & =-\frac{\cos (\theta)\left(e^{\beta} \cos (\phi-\alpha)-\cos (\phi)\right)}{\rho^{2} e^{\beta} \sin ^{3}(\theta)}, \\
\partial_{y} \partial_{\eta^{\prime}} u & =-\frac{\cos (\theta)\left(e^{\beta} \sin (\phi-\alpha)-\sin (\phi)\right)}{\rho^{2} e^{\beta} \sin ^{3}(\theta)}, \\
\partial_{x} \partial_{y^{\prime}} u & =-\frac{\cos (\theta)\left(-e^{\beta} \cos (\phi-\alpha)+\cos (\phi)\right)}{\rho^{2} e^{2 \beta} \sin ^{3}(\theta)}, \\
\partial_{\eta} \partial_{y^{\prime}} u & =-\frac{\cos (\theta)\left(-e^{\beta} \sin (\phi-\alpha)+\sin (\phi)\right)}{\rho^{2} e^{2 \beta} \sin ^{3}(\theta)},
\end{aligned}
$$




$$
\begin{aligned}
& \partial_{y} \partial_{y^{\prime}} u=-\frac{(2 \cosh \beta-\cosh r)}{\rho^{2} e^{\beta} \sin ^{2}(\theta)}, \\
& \partial_{x} \partial_{x^{\prime}} u=-\frac{1}{\rho^{2} e^{\beta} \sin ^{2}(\theta)}, \\
& \partial_{\eta} \partial_{\eta^{\prime}} u=-\frac{1}{\rho^{2} e^{\beta} \sin ^{2}(\theta)}, \\
& \partial_{x} \partial_{\eta^{\prime}} u=\partial_{\eta} \partial_{x^{\prime}} u=0 \text {, } \\
& \partial_{y} u=\frac{1}{\rho \sin \theta}\left(e^{-\beta}-\cosh r\right), \\
& \partial_{x} u=-\frac{\cos (\theta)\left(e^{\beta} \cos (\phi-\alpha)-\cos (\phi)\right)}{\rho e^{\beta} \sin ^{2}(\theta)}, \\
& \partial_{\eta} u=-\frac{\cos (\theta)\left(e^{\beta} \sin (\phi-\alpha)-\sin (\phi)\right)}{\rho e^{\beta} \sin ^{2}(\theta)}, \\
& \partial_{y^{\prime}} u=\frac{1}{\rho \sin \theta e^{\beta}}\left(e^{\beta}-\cosh r\right), \\
& \partial_{x^{\prime}} u=\frac{\cos (\theta)\left(e^{\beta} \cos (\phi-\alpha)-\cos (\phi)\right)}{\rho e^{\beta} \sin ^{2}(\theta)}, \\
& \partial_{\eta^{\prime}} u=\frac{\cos (\theta)\left(e^{\beta} \sin (\phi-\alpha)-\sin (\phi)\right)}{\rho e^{\beta} \sin ^{2}(\theta)} .
\end{aligned}
$$

The mirror image contributions are given by

$$
\begin{aligned}
\partial_{y} \partial_{x^{\prime}} u & =-\frac{\cos (\theta)\left(e^{\beta} \cos (\phi-\alpha)+\cos (\phi)\right)}{\rho^{2} e^{\beta} \sin ^{3}(\theta)} \\
\partial_{y} \partial_{\eta^{\prime}} u & =-\frac{\cos (\theta)\left(e^{\beta} \sin (\phi-\alpha)-\sin (\phi)\right)}{\rho^{2} e^{\beta} \sin ^{3}(\theta)} \\
\partial_{x} \partial_{y^{\prime}} u & =\frac{\cos (\theta)\left(e^{\beta} \cos (\phi-\alpha)+\cos (\phi)\right)}{\rho^{2} e^{2 \beta} \sin ^{3}(\theta)}, \\
\partial_{\eta} \partial_{y^{\prime}} u & =-\frac{\cos (\theta)\left(-e^{\beta} \sin (\phi-\alpha)+\sin (\phi)\right)}{\rho^{2} e^{2 \beta} \sin ^{3}(\theta)}, \\
\partial_{y} \partial_{y^{\prime}} u & =-\frac{(2 \cosh \beta-\cosh r)}{\rho^{2} e^{\beta} \sin ^{2}(\theta)} \\
\partial_{x} \partial_{x^{\prime}} u & =-\frac{1}{\rho^{2} e^{\beta} \sin ^{2}(\theta)}, \\
\partial_{\eta} \partial_{\eta^{\prime}} u & =-\frac{1}{\rho^{2} e^{\beta} \sin ^{2}(\theta)}, \\
\partial_{x} \partial_{\eta^{\prime}} u & =\partial_{\eta} \partial_{x^{\prime}} u=0, \\
\partial_{y} u & =\frac{1}{\rho \sin \theta}\left(e^{-\beta}-\cosh r\right), \\
\partial_{x} u & =-\frac{\cos (\theta)\left(e^{\beta} \cos (\phi-\alpha)+\cos (\phi)\right)}{\rho e^{\beta} \sin ^{2}(\theta)} \\
\partial_{\eta} u & =-\frac{\cos (\theta)\left(e^{\beta} \sin (\phi-\alpha)-\sin (\phi)\right)}{\rho e^{\beta} \sin ^{2}(\theta)} \\
\partial_{y^{\prime}} u & =\frac{1}{\rho \sin \theta e^{\beta}}\left(e^{\beta}-\cosh r\right),
\end{aligned}
$$

\section{APPENDIX B: CALCULATION IN THE DIRICHLET CASE}

Let us consider the case where we impose the Dirichlet B.C. on all the components of all the fields. In this case we can flip the sign of the mirror image contributions in the calculation of the heat kernel. For the scalar part, we get

$$
\begin{aligned}
& S_{\text {scalar }}^{(1)}=\frac{1}{2} \int_{+0}^{\infty} \frac{d t}{t} \int_{\text {thermal AdS }} d^{3} x \sum_{n} \sqrt{g}
\end{aligned}
$$

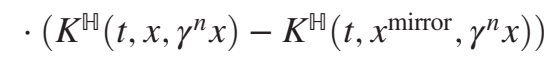

$$
\begin{aligned}
& =\sum_{n=1}^{\infty} \frac{|q|^{n\left(1+\sqrt{m^{2}+1}\right)}}{n\left|1-q^{n}\right|^{2}}-\sum_{n=1}^{\infty} \frac{|q|^{n\left(1+\sqrt{m^{2}+1}\right)}}{n\left(1-|q|^{2 n}\right)} \text {. }
\end{aligned}
$$

The vector and symmetric traceless part can also be calculated,

$$
\begin{aligned}
& S_{\text {vector }}^{(1)}=\int_{0}^{\infty} \frac{d t}{t} \int d^{3} x \sqrt{g} \sum_{n} g^{\mu \rho} \frac{\partial\left(\gamma^{n} x\right)^{\nu^{\prime}}}{\partial x^{\rho}} \\
& \cdot\left(K_{\mu \nu^{\prime}}^{\mathbb{\sharp}}\left(t, r\left(x, \gamma^{n} x\right)\right)-K_{\mu \nu^{\prime}}^{\mathbb{\sharp}}\left(t, r\left(x_{\text {mirror }}, \gamma^{n} x\right)\right)\right) \\
& =\sum_{n=1}^{\infty} \frac{q^{n}+\bar{q}^{n}+|q|^{2 n}}{n\left|1-q^{n}\right|^{2}}-\sum_{n=1}^{\infty} \frac{q^{n}+\bar{q}^{n}+|q|^{2 n}}{n\left(1-|q|^{2 n}\right)},
\end{aligned}
$$

and

$$
\begin{aligned}
S_{s p i n-2}^{(1)}= & \int_{0}^{\infty} \frac{d t}{t} \int d^{3} x \sqrt{g} \sum_{n} g^{\mu \rho} \frac{\partial\left(\gamma^{n} x\right)^{\mu^{\prime}}}{\partial x^{\rho}} g^{\nu \sigma} \frac{\partial\left(\gamma^{n} x\right)^{\nu^{\prime}}}{\partial x^{\sigma}} \\
& \cdot\left(K_{\mu \mu^{\prime}, \nu \nu^{\prime}}^{\llbracket}\left(t, r\left(x, \gamma^{n} x\right)\right)-K_{\mu \mu^{\prime}, \nu \nu^{\prime}}^{\llbracket \natural}\left(t, r\left(x_{\text {mirror }}, \gamma^{n} x\right)\right)\right) \\
= & \sum_{n=1}^{\infty}\left(\frac{1}{n(\cosh \beta-\cos \alpha)}-\frac{1}{n \sinh \beta}\right) \\
& \cdot\left(e^{-\beta} \cos 2 \alpha+e^{-2 \beta} \cos \alpha+\frac{e^{-\sqrt{5} \beta}}{2}\right) .
\end{aligned}
$$

Finally, the gravity partition function becomes

$$
\begin{aligned}
\log Z_{\text {gravity }}^{1-\text { loop }}= & -\log \frac{\sqrt{\operatorname{det} \Delta^{\text {graviton } \operatorname{det} \Delta^{\text {scalar }}}}}{\operatorname{det} \Delta^{\text {vector }}} \\
= & -\sum_{n=2}^{\infty} \frac{\log \left|1-q^{n}\right|^{2}}{2} \\
& -\sum_{n=1}^{\infty} \frac{1}{2 n} \frac{1}{1-|q|^{2 n}}\left(q^{2 n}\left(1-\bar{q}^{n}\right)+\bar{q}^{2 n}\left(1-q^{n}\right)\right) .
\end{aligned}
$$


[1] G. 't Hooft and M. J. G. Veltman, One loop divergencies in the theory of gravitation, Ann. Inst. H. Poincare Phys. Theor. A 20, 69 (1974), https://inspirehep.net/literature/95368.

[2] J. M. Maldacena, The large N limit of superconformal field theories and supergravity, Adv. Theor. Math. Phys. 2, 231 (1998).

[3] S. Giombi, A. Maloney, and X. Yin, One-loop partition functions of 3D gravity, J. High Energy Phys. 08 (2008) 007.

[4] A. Maloney and E. Witten, Quantum gravity partition functions in three dimensions, J. High Energy Phys. 02 (2010) 029.

[5] X. Yin, Partition functions of three-dimensional pure gravity, Commun. Num. Theor. Phys. 2, 285 (2008).

[6] M. Fujita, T. Takayanagi, and E. Tonni, Aspects of AdS/ BCFT, J. High Energy Phys. 11 (2011) 043.

[7] M. Nozaki, T. Takayanagi, and T. Ugajin, Central charges for BCFTs and holography, J. High Energy Phys. 06 (2012) 066.

[8] T. Takayanagi, Holographic Dual of BCFT, Phys. Rev. Lett. 107, 101602 (2011).

[9] A. Karch and L. Randall, Open and closed string interpretation of SUSY CFT's on branes with boundaries, J. High Energy Phys. 06 (2001) 063.

[10] I. G. Moss and P. J. Silva, BRST invariant boundary conditions for gauge theories, Phys. Rev. D 55, 1072 (1997).

[11] P. van Nieuwenhuizen and D. V. Vassilevich, Consistent boundary conditions for supergravity, Classical Quantum Gravity 22, 5029 (2005).

[12] R.-X. Miao, Holographic BCFT with dirichlet boundary condition, J. High Energy Phys. 02 (2019) 025.

[13] P. Kraus, Lectures on black holes and the AdS(3)/CFT(2) correspondence, Lect. Notes Phys. 755, 193 (2008).

[14] R. Dijkgraaf, J. M. Maldacena, G. W. Moore, and E. P. Verlinde, A black hole Farey tail, arXiv:hep-th/0005003.

[15] M. Banados, M. Henneaux, C. Teitelboim, and J. Zanelli, Geometry of the $(2+1)$ black hole, Phys. Rev. D 48, 1506 (1993).

[16] S. Carlip and C. Teitelboim, Aspects of black hole quantum mechanics and thermodynamics in $(2+1)$-dimensions, Phys. Rev. D 51, 622 (1995).
[17] G. W. Gibbons and S. W. Hawking, Action integrals and partition functions in quantum gravity, Phys. Rev. D 15, 2752 (1977).

[18] J. M. Maldacena and A. Strominger, AdS(3) black holes and a stringy exclusion principle, J. High Energy Phys. 12 (1998) 005.

[19] J. R. David, M. R. Gaberdiel, and R. Gopakumar, The heat kernel on $\operatorname{AdS}(3)$ and its applications, J. High Energy Phys. 04 (2010) 125.

[20] R. Gopakumar, R. K. Gupta, and S. Lal, The heat kernel on AdS, J. High Energy Phys. 11 (2011) 010.

[21] R. B. Mann and S. N. Solodukhin, Quantum scalar field on three-dimensional (BTZ) black hole instanton: Heat kernel, effective action and thermodynamics, Phys. Rev. D 55, 3622 (1997).

[22] D. V. Vassilevich, Heat kernel expansion: User's manual, Phys. Rep. 388, 279 (2003).

[23] I. Akal, Y. Kusuki, T. Takayanagi, and Z. Wei, Codimension two holography for wedges, Phys. Rev. D 102, 126007 (2020).

[24] V. Balasubramanian and P. Kraus, A stress tensor for Anti-de Sitter gravity, Commun. Math. Phys. 208, 413 (1999).

[25] E. D'Hoker, D. Z. Freedman, S. D. Mathur, A. Matusis, and L. Rastelli, Graviton and gauge boson propagators in $\operatorname{AdS}(d+1)$, Nucl. Phys. B562, 330 (1999).

[26] E. Witten, A note on boundary conditions in Euclidean gravity, Rev. Math. Phys. 33, 2140004 (2021).

[27] N. Benjamin, H. Ooguri, S.-H. Shao, and Y. Wang, Lightcone modular bootstrap and pure gravity, Phys. Rev. D 100, 066029 (2019).

[28] N. Benjamin, S. Collier, and A. Maloney, Pure gravity and conical defects, J. High Energy Phys. 09 (2020) 034.

[29] J. Polchinski, String Theory. Vol. 1: An Introduction to the Bosonic String, Cambridge Monographs on Mathematical Physics (Cambridge University Press, Cambridge, England, 2007).

[30] J. M. Maldacena and L. Maoz, Wormholes in AdS, J. High Energy Phys. 02 (2004) 053. 